\title{
Vildagliptina en pacientes diabéticos tipo 2 con riesgo cardiovascular o compromiso renal. Revisión de literatura.
}

Victor Hugo Forero Supelano1, Gabriel German Ribón Quintero', Jairo Echeverry-Raad ${ }^{3}$

\footnotetext{
1 Medicina Familiar Integral, Magister Epidemiología Clínica - Profesor Titular Fundación Universitaria Clínica Juan N. Corpas. Dirección Investigación Clínica Juan N. Corpas.

2 Médico Internísta, Medical Manager Cardiovascular y Metabólica, Novartis Andina.

3 Profesor Titular Facultad de Medicna Univesridad Nacional de Colombia. Docente Investigador Fundación Universitaria Juan N. Corpas.
}

Autor de correspondencia: Víctor Hugo Forero Supelano. victor. forero@juanncorpas.edu.co

Como citar: Forero VH, Ribon GG, Echeverry-Raad J. Vildagliptina en pacientes diabéticos tipo 2 con riesgo cardiovascular o compromiso renal. Revisión de literatura. Revista Cuarzo 2018:24 (1) 28-45.

Recibido: 21 de mayo de 2018 Aceptado: 25 de junio de 2018 Publicado: 30 de junio de 2018

\section{Fuente ayuda}

La presente revisión de literatura da continuidad a la Revisión Sistemática (literatura gris) de 19 ECCAs publicados entre 2008 y 2015 sobre efectividad y seguridd con el DPP-4I vildagliptina en diabetes mellitus tipo 2, que derivó en proceso continuado (living review), de análisis crítico de evidencias promovido y soportado por Novartis SA.

La interpretación de los datos y los puntos de vista que sobre los mismos expresamos los autores son de nuestra entera responsabilidad.

\section{Resumen}

Introducción: los fármacos que se prescriben comúnmente para el tratamiento de la diabetes mellitus tipo 2 (DM2) tienen restricciones de uso en pacientes con deterioro cardiovascular o renal. Estudios publicados sobre el grupo farmacológico inhibidores de dipeptidil peptidasa-4 (DPP-4I) indican que se trata de agentes efectivos y seguros para su uso en esta población de pacientes. Sin embargo, en la práctica clínica cotidiana, prevalecen vacíos e incertidumbre respecto de la efectividad y seguridad para prescribir apropiadamente los DPP-4I, específicamente en pacientes en quienes concurre el riesgo o presencia de enfermedad cardiovascular o renal.

Esta revisión de literatura integra estudios publicados entre 2015-2018, en seguridad, eficacia y uso de vildagliptina para pacientes con DM2 en riesgo incrementado de eventos o presencia de enfermedad cardiovascular, función renal comprometida, o ambos.

Método: revisión de literatura publicada desde 01-01-2015 a 31-05-2018, enfocada en seguridad, eficacia y uso de vildagliptina en pacientes adultos con DM2 en riesgo de evento o presencia de enfermedad cardiovascular o con compromiso de la función renal, o ambos.

Resultados: conjunto de evidencias de tres estudios de vida, seis ECCA, dos meta-análisis en red, nueve revisiones sistemáticas y tres estudios observacionales integran la unidad de análisis que presenta en síntesis narrativa desenlaces de efectividad y seguridad del DPP-4I vildagliptina en el contexto de pacientes diabéticos con riesgo cardiovascular y compromiso de función renal.

Conclusión: esta revisión reitera la trascendencia de intervenciones integrales, efectivas y seguras, para el control metabólico en personas con DBT, específicamente para pacientes en quienes concurre enfermedad cardiovascular, deterioro de función renal, y esquemas combinados con hipoglucemiante oral. En este contexto vildagliptina reúne valiosa evidencia de efectividad y seguridad que permite apoyar decisiones clínicas sustentadas. A destacar los beneficios para pacientes de edad avanzada, grupo especialmente frágil y vulnerable a eventos cardiovasculares.

Palabras clave: Diabetes mellitus, efectividad, seguridad, riesgo cardiovascular, vildagliptina.

Licencia creative (c) (1) ()

commons 


\title{
Vildagliptin in diabetic type 2 patients with cardiovascular risk or renal impairment. Review of the literatura
}

\begin{abstract}
Introduction: Commonly prescribed agents for the treatment of type 2 diabetes (T2DM) have restrictions on use in patients with cardiovascular or renal function impairment. Published studies on dipeptidyl peptidase-4 inhibitors (DPP-4I) indicate that these agents are suitable for use in this patient population. However, in everyday clinical practice, uncertainties prevail regarding the effectiveness and safety of appropriately prescribing the DPP-4I, specifically in patients in whom the risk or presence of cardiovascular or renal disease is present.
\end{abstract}

This literature review integrates studies published between 2015 -2018 on safety, efficacy and use of vildagliptin in patients with DM2 at risk of events or cardiovascular disease, impaired renal function, or both.

Method: literature review that extends from 01-01- 2015 to 31-05-2018, focuses on the safety, efficacy and clinical use of vildagliptin in adult patients with DM2 at risk of event, or cardiovascular disease, or impaired renal function, or both.

Results: three real world data studies, six ECCA, two network meta-analyses, nine systematic reviews, and three observational studies integrate the unit of analysis that presents in narrative synthesis on the effectiveness and safety of the DPP-4I vildagliptin in the context of diabetic patients with cardiovascular risk and impaired renal function.

Conclusion: this review reiterates the transcendence of comprehensive, effective and safe interventions for metabolic control in people with DBT, specifically for patients in whom cardiovascular disease, deterioration of renal function, and combined schemes with oral hypoglycemia. In this context vlidagliptin gathers valuable evidence on effectiveness and safety that allows supporting sustained clinical decisions. To highlight the benefits for elderly patients, a particularly fragile group and vulnerable to cardiovascular events.

Keywords:Diabetes mellitus, efficacy, safety, cardiovascular risk, vildagliptin

\section{Introducción}

Común, crónica y con impacto negativo en la calidad de vida y sobrevida son aspectos que caracterizan a la diabetes mellitus (DBT). Se calcula que habrá cerca de 642 millones de personas con diabetes en 2040. Particularmente durante las dos últimas décadas crece evidencia confirmando que, junto al infarto agudo de miocardio y diversas manifestaciones ateroescleróticas, la falla cardíaca es un problema de morbilidad y mortalidad preponderante en los pacientes con DBT.

Entre los estudios más sobresalientes para determinar la relación entre DBT y enfermedad cardiovascular - falla cardíaca - está la cohorte retrospectiva Kaiser Permanente Northwest database (1) con 8231 pacientes diabéticos sin falla cardíaca, emparejados por edad y sexo con 8845 sujetos no diabéticos. En el seguimiento de 6 años, la incidencia de falla cardíaca en el grupo de diabéticos fue de 30.9/100.000 personas año, versus 12.4/100.000 personas año en el grupo de no diabéticos.

Algo más, el compromiso de la función renal en los pacientes diabéticos es la principal causa de enfermedad renal crónica $(2,3)$; lo que a su vez incrementa el riesgo de eventos cardiovasculares y muerte asociada; no obstante los beneficios de la insulinoterapia y demás intervenciones en procura del mejoramiento y recuperación posible de la salud de esta población. Así es que concurren; entre otros factores no menos importantes, el desorden metabólico, la enfermedad cardiovascular y el compromiso renal configurando el espectro de comorbilidad y riesgos superpuestos a la ya deteriorada calidad de vida en una persona con diabetes.

En este contexto resulta obligado analizar las evidencias existentes respecto de las implicaciones fármaco-terapéuticas para todos los grupos de medicamentos dirigidos al control metabólico en DBT.

En los últimos años, los inhibidores de dipeptidil peptidasa-4 (DPP-4I), han integrado al conjunto de opciones terapéuticas de los hipoglucemiantes orales, entre otras razones, gracias a su efecto sobre la dipeptidil peptidasa 4 (DPP4), enzima multifuncional que puede inactivar las incretinas, incluido el glucagón péptido 1 (GLP-1) y péptido inhibidor gástrico (GIP), para privar sus efectos anti-hiperglucemiantes (4).Cuando se usan inhibidores de DPP-4I, el conjunto disponible de GLP-1 activo y GIP puede ser restaurado disponiendo a un control glucémico que se ejerce de manera dependiente de la glucosa. Vildagliptina, potente y selectivo inhibidor de DPP4, promueve y facilita el control glicémico al aumentar la disponibilidad de incretinas endógenas, potencia la secreción de insulina dependiente de la glucosa y suprime la liberación de glucagón.

Específicamente para vildagliptina en monoterapia y en combinación (add-on) con metformina, o asociada a otros hipoglucemiantes orales y con insulina, se destacan numerosos estudios de 
comprobada efectividad y seguridad (5-11), evidencia que se ha incorporado a las recomendaciones en guías de práctica clínica en DM2 (12).

Esta revisión integra y sintetiza evidencias, publicadas desde 2015 a 2018, con el propósito de analizar eficacia y seguridad del DPP-4I; vildagliptina, en comparación con otros hipoglucemiantes orales en adultos con diabetes mellitus tipo 2 (DM2), con comorbilidad cardiovascular, renal o ambas. Igualmente presenta información relacionada con el uso concurrente de vildagliptina en pacientes bajo tratamiento con insulina.

\section{Método}

Bajo lineamientos de revisión rápida-(Rapid Review) $(13,14)$; modelo que permite reducir el tiempo que demanda el proceso metodológico regular de revisión sistemática (RS), y no obliga al intento sumario meta-analítico, los estudios fueron cualificados para exponerlos de manera independiente pero integrativa, de lo que deriva una síntesis narrativa de evidencias en efectividad (niveles de HbAlc alcanzada) y seguridad (eventos hipoglucémicos, eventos cardiovasculares) por comparaciones del DPP-4I vildagliptina en el tratamiento de DM2 en personas de 18 años o más y en quienes coexiste alguna de las siguientes condiciones: enfermedad cardiovascular - riesgo cardiovascular - compromiso de función renal (cualquier grado), pacientes en insulinoterapia.

La estrategia de búsqueda incluyó términos texto libre y $\mathrm{MeSH}$ que se combinaron para "diabetes mellitus, type2", "renal disease", "chronic kidney disease", "renal failure", "albuminuria", "renal impairment", "heart failure", "cardiovascular risk", "myocardial infarction", junto con "DPP-4 inhibitor", "gliptin", "vildagliptin", "efficacy", "safety". Extendidos con límites período enero 01-2015 a diciembre 31-2018, idioma Inglés y español, para ensayos clínicos controlados (ECCA), y estudios agregativos. Última consulta 31-05-18.

Ante los reiterados cuestionamientos sobre la transferibilidad de resultados de los ECCA a la práctica clínica; concretamente por el estricto criterio de selección de pacientes y el relativamente corto tiempo de seguimiento que pudiere haber en algunos estudios, fueron analizados e incluidos; en función de su calidad, estudios de vida real (Real World Data).

Las bases de datos utilizadas fueron MEDLINE/PUBMED (Plataforma OVID), EMBASE (Plataforma EBSCOhost - Elsevier), Clinical Trials (Clinical Trials.gov, OVID), Cochrane Database of Systematic Reviews (Plataforma Wiley).

En la valoración de la calidad de las RS que incluyesen estudios randomizados y no-randomizados nos apoyamos en los 16 criterios AMSTARD-2 (15). Para estudios primarios seguimos los lineamientos de evaluación crítica y metodológica de los ensayos clínicos de Downs y Black (16), herramienta con lista de verificación probadamente confiable en la valoración de calidad, validez interna y externa, tanto para ECCAs como para estudios no aleatorizados.

Luego de realizar la búsqueda de información en las bases de datos con los criterios predefinidos, filtramos y seleccionamos los estudios con base en los siguientes elementos de juicio:

- Estudios en los que fuese evidente y verificable la minimización de sesgos: enmascaramiento, declaración de conflictos, revisión por pares y accesibilidad.

- Desenlace atribuible al efecto de la intervención (control de variables de confusión): aleatorización y asignación a grupo intervención y grupo comparador, mayor tiempo de observación y seguimiento a los desenlaces de efectividad y seguridad, significancia clínica y estadística, especial atención en diferencias clínicamente importantes

- Representatividad de los grupos de pacientes incluidos en los estudios: verificación de criterios de inclusión-exclusión, características generales de la población, potencial para la transferencia de los resultados a la práctica clínica en contextos fuera de proceso de investigación. Consistencia entre el conjunto de evidencias.

- Reproducibilidad de la magnitud y direccionalidad los estimadores para los desenlaces dentro de los diferentes estudios.

\section{Resultados}

Un conjunto de 23 estudios en total: 3 estudios de vida real, 6 ECCA, 2 Meta-análisis en red, 9 revisiónes sistemáticas y 3 estudios observacionales componen la unidad de análisis final. El ordenamiento en la presentación y síntesis obedece a la fecha descendente de publicación; con independencia del tipo de estudio.

Por considerarlo relevante $\mathrm{v}$ conveniente. hemos aprovechado datos provenientes ECCAs publicados con posterioridad a 2008 y antes de 2015 junto a los cuales es posible contrastar y realizar análisis de efectividad y seguridad de vildagliptina en riesgo cardiovascular o disfunción renal cuando se el caso. Lo mismo anlica con anuellos estudios de vida real nara identificar cuán cercanos o distantes pudieren ser los resultados de efectividad reportados en los ECCA.

Para brindar un contexto general de los resultados de efectividad - cambio HbA1c - y seguridad con vildagliptina; exponemos en primer lugar el sumario de 4 estudios sin restricción o especificación a riesgo cardiovascular, compromiso de función renal o tratamiento con insulina. Posteriormente queda expuesta una síntesis de 10 estudios que evaluaron efectividad y seguridad de vildagliptin en pacientes con DM2 en el contexto de riesgo cardiovascular, 3 estudios en pacientes DM2 con compromiso de función renal y finalmente 6 estudios relacionados con los desenlaces de efectividad y seguridad de vildagliptina mas insulina. 
Contexto general de los resultados de efectividad - seguridad con vildagliptina

Cohen 2018 (17) - Estudio de vida real en 345 pacientes en tratamiento con metformina, sin control óptimo y en quienes se evaluó el resultado de adicionar vildagliptina para control metabólico a 90 días y el efecto en peso corporal.

Las características generales de los pacientes incluidos en el análisis corresponden a personas con edad media de 60 años, con diabetes de más de 10 años de evolución para la tercera parte de la población, $58 \%$ hombres.

En media, la hemoglobina glucosilada (HbA1c) fue $0.9 \%$ menor de manera clínicamente significativa (IC95 \% - 0.7 - -1.0\%), y asociada de manera directa con la hemoglobina glucosilada basal.
Estos resultados de vida real son semejantes a los reportados en el análisis pos-hoc del ECCA que comparó la adición de vildagliptina versus sulfonilureas a metformina en pacientes en quienes no se lograba control metabólico (18).

Wang 2017 (19) - Meta-análisis en red- permite comparar en forma simultánea diferentes intervenciones- de 8 ECCAs. Este estudio reunió 3180 pacientes para identificar el cambio en $\mathrm{HbA1c}$, los niveles de triglicéridos y eventos adversos entre 7 esquemas diferentes: (placebo + metformina), (dapagliflozina + metformina), (vildagliptina + metformina), (saxagliptina + metformina), (empaglifozina + metformina), (exenatida + metformina) y (sitagliptina + metformina).

Los desenlaces comparados entre las diferentes combinaciones son expuestos en la tabla 1 Wang 2017

Tabla I. Wang 2017- Cambio en HbA1c, glucemia, lípidos, peso corporal

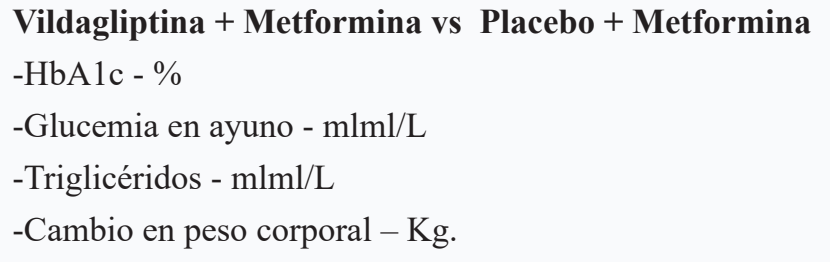

$-1.95(-3.70--0.23)$

$-2.42(-5.29-0.27)$

$-0.70(-1.62-0.23)$

$-0.24(-3.31-2.57)$

$\begin{array}{lr}\text { Vildagliptina + Metformina vs Dapaglifozina + Metformina } & \\ \text {-HbAlc \% } & -1.43(-3.63-0.59) \\ \text {-Glucemia en ayuno - mlml/L } & -1.38(-4.81-1.93) \\ \text {-Triglicéridos ml/L } & -0.73(-5.41-3.87) \\ \text {-Cambio en peso corporal- Kg } & 1.63(-2.62-6.14)\end{array}$

Vildagliptina + Metformina vs Exenatide + Metformina

$-\mathrm{HbA} 1 \mathrm{c} \%$

$-1.55(-4.13-0.93)$

-Glucemia en ayuno - $\mathrm{mlml} / \mathrm{L}$

$-2.11(-6.16-1.80)$

-Triglicéridos $\mathrm{ml} / \mathrm{L}$

-Cambio en peso corporal- Kg

$-1.36(-2.64--0.01)$

No dato

$\begin{array}{lc}\text { Vildagliptina + Metformina vs Sitagliptina + Metformina } & \\ \text {-HbAlc } \% & -1.32(-3.79-1.21) \\ \text {-Glucemia en ayuno - mlml/L } & -1.13(-5.15-2.69) \\ \text {-Triglicéridos ml/L } & \text { No dato } \\ \text {-Cambio en peso corporal- Kg } & \text { No dato }\end{array}$

Con base en las comparaciones realizadas para siete esquemas terapéuticos este estudio identifica, en los valores de la superficie bajo la curva - surface under the cumulative ranking curve - (SUCRA), a la combinación vildagliptina+metformina con el mayor puntaje de control metabólico global. Se ubica como primera opción en reducción de HbA1c con valor 0.96, para control de glucemia en ayuno 0.91 , para control colesterol total 0.91 , triglicéridos 0.88 y en tercer lugar para control de peso 0.50 , datos en tabla 2 Wang 2017. 


\begin{tabular}{|c|c|c|c|c|c|}
\hline Combinación & HbA1c & Glucemia ayuno & Colesterol & Triglicéridos & $\begin{array}{c}\text { Control de } \\
\text { peso }\end{array}$ \\
\hline Placebo + Metformina & 0.27 & 0.31 & 0.58 & 0.62 & 0.42 \\
\hline Dapaglifozina + Metformina & 0.59 & 0.63 & 0.55 & 0.62 & 0.79 \\
\hline Saxagliptina + Metformina & No dato & No dato & No dato & No dato & No dato \\
\hline Empaglifl. + Metformina & No dato & No dato & No dato & No dato & No dato \\
\hline
\end{tabular}

Esposito 2015 (20) - Revisión sistemática y meta-análisis de 78 ensayos clínicos, aporta evidencia para estimar los resultados esperados de cambio en $\mathrm{HbA1c}$, gracias al nomograma (figura 1) construido con base en información obtenida en más de 24000 pacientes diabéticos tipo 2, y que permite estimar la efectividad comparativa entre diferentes opciones de DPP-4I.

Figura I (adaptada). Nomograma predictivo de eficacia en disminución de HbAlc comparativa entre los diversos DPP-4I.

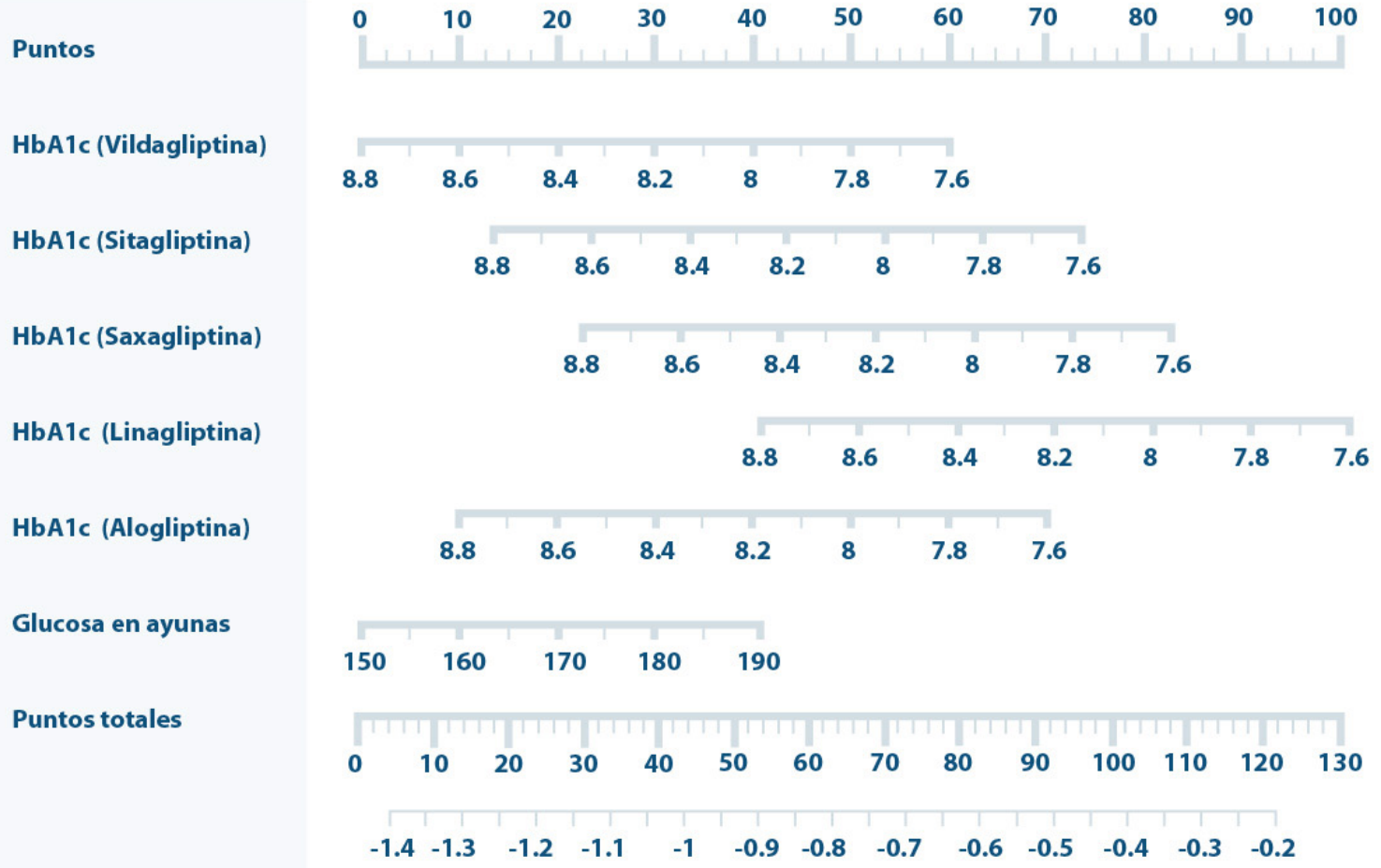

Para utilizar el nomograma el primer paso es elegir uno de los inhibidores, identificar la HbA1c basal y la glucosa en ayunas. Desde esos puntos se proyectan líneas perpendiculares a la escala de puntos (superior). Sumar los valores que señalen las perpendiculares y ese valor proyectarlo sobre la escala de "Puntos totales" para encontrar el valor de HbAlc esperado por la administración de inhibidor DPP4 elegido. 
Godoy 2015 (21) - Estudio de vida real en 115 chilenos adultos con DM2 para evaluar el control glicémico, seguridad y tolerancia a vildagliptina, quienes bajo tratamiento con combinación de dos hipoglucemiantes no habían logrado control óptimo.

Los niveles de $\mathrm{HbA} 1 \mathrm{c}$ promedio basales fueron - en media - 8,3 $\pm 1,4 \%$, luego de 12 semanas de tratamiento con vildagliptina la HbA1c descendió a 7,2 $\pm 1,1 \%$. Previo al cambio a vildagliptina la proporción de pacientes con $\mathrm{HbA} 1 \mathrm{c}<6,5$ era $2 \%$ y $\mathrm{HbA} 1 \mathrm{c}<7$, era $15 \%$.

Con vildagliptina dichas proporciones se incrementaron al 25\% y $52 \%$ respectivamente, esto acompañado de reducción notable y clínicamente relevante respecto de los eventos de hipoglucemia: $22 \%$ cayó a $3 \%(\mathrm{p}<0.00001)$, y a destacar dicha diferencia cuando se analiza para mayores de 60 años de edad en quienes bajo el tratamiento previo los episodios contabilizaron $28 \%$ versus 3\% cuando recibieron vildagliptina, datos consistentes con los reportados en el estudio EDGE (22), uno de los estudios con datos de vid real más completos por el número de pacientes -45.000-, de 27 países en cinco regiones.

\section{Vildagliptina en riesgo cardiovascular}

El debate sobre la seguridad cardiovascular con terapia basada en incretinas creció y fue más visible en el hallazg inesperado de incremento de $27 \%$ en riesgo para hospitalización por insuficien- cia cardiaca en el estudio SAVOR TIMI 53 (23). Esto, además de nutrir el debate, alentó la hipótesis de exacerbación o precipitación de falla cardíaca con los DPP-4I y motivó la búsqueda activa de desenlaces cardiovasculares adversos en pacientes que recibieran este grupo de hipoglucemiantes, para el efecto los estudios TECOS (sitagliptina) (24), EXAMINE (alogliptina) (25), CARMELINA (linagliptina) (26), VIVIDD (vildagliptina) (27), este último, a diferencia de los cuatro anteriores, se diseñó específicamente caracterizando los pacientes de acuerdo a la presencia (basal) de falla cardiaca o identificando el desarrollo de la falla cardíaca durante el seguimiento, además de determinar la función ventricular izquierda.

McMurray 2018 (27) - ECCA fase IV de No inferioridad - doble enmascarado en 254 adultos DBT 2, con fracción de eyección $<40 \%$, (en NYHA I-III), para evaluar como primer desenlace principal el efecto atribuible a vildagliptina (add-on tratamiento estándar) en la función ventricular izquierda (VIVIDD). En este estudio participaron 67 centros de 15 países.

La hipótesis evaluada en este estudio plantea que vildagliptina es al menos no inferior que placebo para el cambio en la fracción de eyección ventricular izquierda (punto de corte predefinido para no inferioridad $-3.5 \%$, p 0.025 ), desde basal a 52 semanas de observación. El segundo desenlace principal a evaluar fue el cambio en HbA1c a 16 semanas. Ver tablas 3 y 4 - McMurray 2018 a y b.

Tabla 3 - McMurray 2018 a - Desenlace para fracción de eyección y cambio en HbA1c

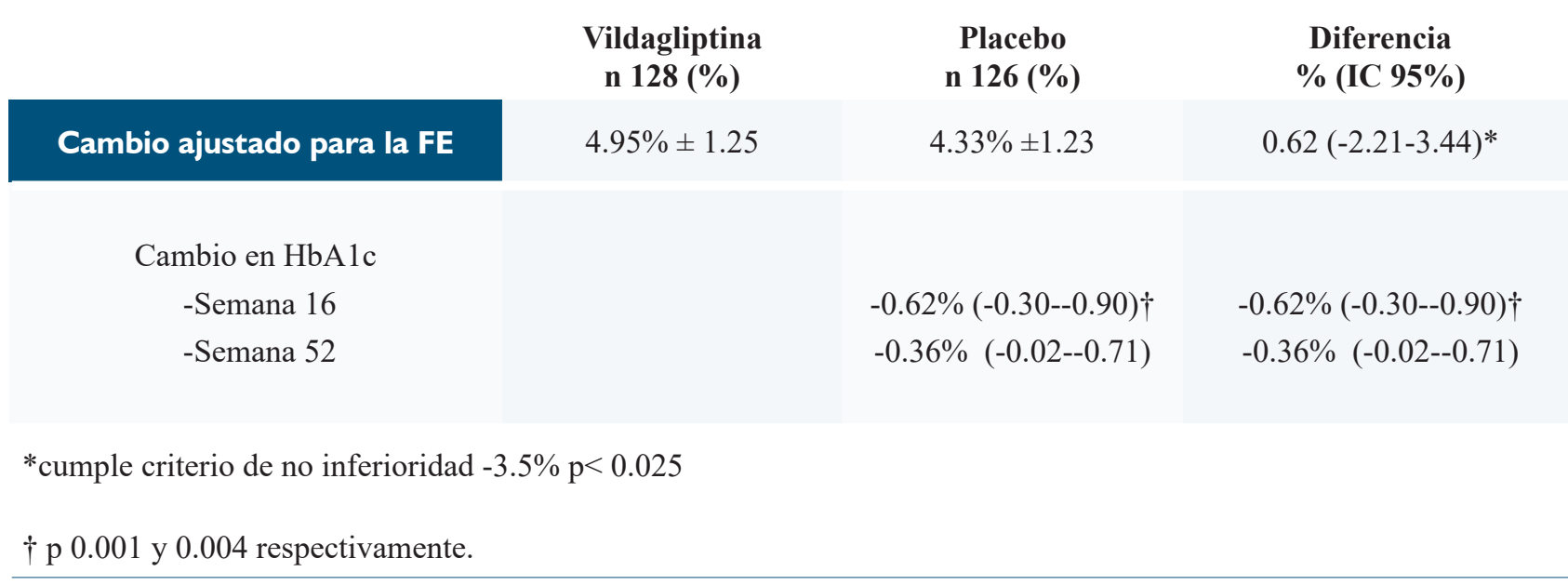

FE: fracción de eyección, HbA1c: hemoglobina glucosilada, IC: intervalo de confianza

La clase funcional NYHA no presento variaciones sustanciales en ninguno de los dos grupos y los ajustes de medicamentos para tratamiento de la falla cardíaca fueron realizados en el $26 \%$ y $24 \%$ para el grupo de vildagliptina y placebo respectivamente (p0.64). 
Tabla 4 - McMurray 2018 b Eventos no fatales y muertes por causa cardiovascular y toda causa

\begin{tabular}{|c|c|c|c|}
\hline & $\begin{array}{c}\text { Vildagliptina } \\
\text { n I } 28(\%)\end{array}$ & $\begin{array}{c}\text { Placebo } \\
\text { n I26 (\%) }\end{array}$ & $\begin{array}{c}\text { Diferencia } \\
\%(I C 95 \%)\end{array}$ \\
\hline Cualquier evento cardiovascular (fatal o no fatal) & $35(27.3)$ & $31(24.6)$ & $2.7(-9.5-15.0)$ \\
\hline Evento vascular cerebral & $1(0.8)$ & $4(17.5)$ & $0.5(-11.9-12.7)$ \\
\hline Empeoramiento de la falla & $23(18.0)$ & $22(17.6)$ & $0.5(-11.9-12.7)$ \\
\hline Sind coronario agudo & $7(5.5)$ & $3(2.4)$ & $3.1(-9.5-15.4)$ \\
\hline Arritmia & $9(7.0)$ & $4(3.2)$ & $3.9(-8.7-16.1)$ \\
\hline Muerte causa cardiovascular & $7(5.5)$ & $4(3.2)$ & $2.3(-10.3-14.6)$ \\
\hline Muerte toda causa & $11(8.6)$ & $4(3.2)$ & $5.4(-7.2-17.6)$ \\
\hline
\end{tabular}

Cuatro de las muertes que se presentaron en el grupo vildagliptina fueron por: neoplasia hepática, neoplasia pulmonar, choque séptico y obstrucción intestinal por fenómeno adherencial peritoneal.

Elgendy 2017 (28) -Revisión sistemática y meta-análisis con la que se aborda la controversial y, en algunos casos, contraria evidencia con la que distintos ECCAs exponen resultados relativos a la seguridad cardiovascular de los DPP-4I.

El estudio reúne evidencias de 66730 pacientes en 90 ECCAs de comparaciones realizadas entre DPP-4I y placebo cuyo desenlace principal a un tiempo medio de 108 semanas fue falla cardíaca representado por un OR 1.11; (IC95\% 0.99-1.25) $\mathrm{I}^{2}=$ $0 \%$. En segundo lugar evento cerebrovascular isquémico OR 0.99; (IC95\% 0.85-1.15), no significativo, mortalidad por toda causa OR 1.03; (IC95\% 0.94-1.12), y por causa cardiovascular OR 1.02; (IC95\% 0.92-1.14), no significativo.

El análisis pre-especificado incluyendo solo aquellos estudios con alta calidad produjo resultados semejantes OR 1.13, (IC95\% 0.99-1.28), $\mathrm{p}=0.07, \mathrm{I}^{2}=27 \%$.

Es interesante notar que en este meta-análisis sus autores atribuyen cualquier potencial incremento de riesgo para falla cardíaca a los datos que provienen del estudio que incluyo el DPP4I saxagliptina SAVOR TIMI 53 (23), en el que se identificó; en análisis post hoc, que el incremento de $27 \%$ del riesgo para hospitalización por insuficiencia cardiaca lo aportaron aquellos pacientes con niveles basales elevados de péptido natriurético, antecedente de falla cardíaca o enfermedad renal crónica.

Fadini 2017 (29) - Estudio observacional retrospectivo que aprovecha las propiedades de una base nacional italiana, sistematizada para propósitos de monitorización y análisis en el uso (vida real) de medicamentos para apoyar toma de decisiones.
Este estudio reúne información lograda en 127555 pacientes bajo tratamiento con DPP-4I en quienes se determinó bajo riesgo a hospitalización por falla cardíaca. Con base en dicha cohorte inicial se realiza análisis intraclase para 17615 pacientes quienes recibían sitagliptina (60\%), vildagliptina (27\%) y saxagliptina (13\%) y se evaluó el riesgo para falla cardíaca en comparación con hipoglucemiantes del tipo sulfonilureas en monoterapia o en combinación con metformina.

En un período de observación de 2.2 años la incidencia de hospitalización por falla cardíaca fue 2.7 episodios por 1000 personas año en el grupo de sitagliptina, 1.7 episodios por 1000 personas año en el grupo de vildagliptina. En términos de número de eventos de hospitalización por falla cardíaca 4.0 eventos por 1000 personas año y 2.1 por 1000 personas año saxagliptina, vildagliptina respectivamente.

La información para el grupo de pacientes bajo tratamiento con sulfonilureas permite identificar tasa global de hospitalización por falla cardíaca de 6.4 eventos por 1000 personas año para quienes recibían glibenclamida y 5.3 por 1000 personas año en glimepirida. Las frecuencias de primera hospitalización por falla cardíaca en quienes recibieron glicazida y glimepirida 4.7 eventos por 1000 personas año y 4.0 por 1000 personas año respectivamente.

Guo 2017 (30) - Meta-análisis en red con 50 ECCA en pacientes con DBT 2, cuya intervención a evaluar/comparar fuese DPP-4I (alogliptina, linagliptina, sitagliptina, saxgliptina y vildagliptina) otros hipoglucemiantes orales (metformina, glipizida, gliclazida, glimepirida, canaglifozina, empaglifozina, pioglitazona, albiglutida, y liraglutida) o placebo para el desenlace de admisión por falla cardíaca.

Al analizar el riesgo de falla cardíaca de un DPP-4I contra placebo resulto significativamente mayor para alogliptina: RR 2.13; 
(IC95\% 1.06-6.26), con similar magnitud y direccionalidad con linagliptina RR 2.76; (IC95\% 0.98-8.31), a diferencia de lo resultante para sitagliptina: RR 0.86; (IC95\% 0.43-1.57), saxagliptina RR 0.84; (IC95\% 0.33-1.61) y más bajo aún con vildagliptina: RR 0.71; (IC95\% CI 0.25-1.68).

Al analizar el riesgo por comparación entre diferentes DPP-4I, es notable el menor riesgo con vildagliptina: alogliptina versus vildagliptin RR 3.05; (IC95\% 1.01-14.19), linagliptina versus vildagliptina RR 3.95; (IC95\% 1.07-17.45), sitagliptina versus vildagliptina RR 1.21; (IC95\% 0.44-3.69) y saxagliptina versus vildagliptina RR 1.18; (IC95\% 0.36-3.57). Frente a alogliptina el análisis de resultados agrupados identifica a vildagliptina con el menor riesgo RR 0.33; (IC95\% 0.07-0.99) le sigue sitagliptina RR 0.40; (IC 95\% 0.11-0.96).

Ahora, al comparar con linagliptina, vildagliptina arrojó un RR 0.25; (IC95\% 0.06-0.94), con sitagliptina RR 0.31; (IC95\% 0.09-0.95), y con saxagliptina RR 0.30; (IC95\% 0.07-0.97).

Vildagliptina registra la más alta probabilidad (49\%) en la clasificación de opción segura en relación con menor riesgo asociado para falla cardíaca, seguida por saxagliptina (27\%). El ordenamiento completo se aprecia en la tabla 5 Guo 2017.

Tabla 5- Guo 2017 Clasificación probabilidad terapéutica entre DPP-4I

\section{Clasificación de probabilidad terapéutica}

\begin{tabular}{|c|c|c|c|c|c|c|c|}
\hline DPP-4I & 1 & 2 & 3 & 4 & 5 & 6 & 7 \\
\hline Alogliptina & $0.12 \%$ & $0.43 \%$ & $1.20 \%$ & $4.86 \%$ & $45.9 \%$ & $27.1 \%$ & $20.4 \%$ \\
\hline Linangliptina & $0.25 \%$ & $0.72 \%$ & $1.2 \%$ & $2.9 \%$ & $23.8 \%$ & $24.6 \%$ & $46.5 \%$ \\
\hline Placebo & $3.13 \%$ & $16.9 \%$ & $38.7 \%$ & $38.9 \%$ & $2.19 \%$ & $0.07 \%$ & $0 \%$ \\
\hline Sitagliptina & $20.7 \%$ & $32.1 \%$ & $26.1 \%$ & $18.7 \%$ & $2.1 \%$ & $0.14 \%$ & $0 \%$ \\
\hline Saxagliptina & $26.5 \%$ & $28.4 \%$ & $19.8 \%$ & $20.8 \%$ & $3,9 \%$ & $0.33 \%$ & $0.03 \%$ \\
\hline Vildagliptina & $49.1 \%$ & $21.3 \%$ & $12.9 \%$ & $13.3 \%$ & $2.7 \%$ & $0.38 \%$ & $0.05 \%$ \\
\hline
\end{tabular}

Williams 2017 (31) -Estudio de base poblacional, analítico, cohorte - datos de vida real - en 5 países de Europa (Alemania, Dinamarca, Francia, Reino Unido, Suecia) para evaluar y comparar vildagliptina (en monoterapia o en combinación con metformina) versus otros antidiabéticos orales en seguridad cardiovascular: infarto de miocardio, evento coronario agudo, evento cerebro-vascular, falla cardíaca (como eventos aislados) o en combinación (desenlace compuesto).

El 2,8 \% de los pacientes (738054) registrados en la multi-base se encontraban bajo tratamiento con vildagliptina, bajo un tiempo de seguimiento de 1.4 años, lo cual resulta en una exposición acumulada a vildagliptina equivalente a 28330 pacientes año. En la tabla 6 Williams 2017 se resumen las tasas de riesgo para cada uno de los eventos cardiovasculares evaluados.

Tabla 6 - Williams 2017-Incidencia de riesgo cardiovascular vildagliptina comparada con otro antidiabético oral

Vildagliptina versus otro Antidiabético oral Incidencia ajustada (IC 95\%)

\section{IAM}

ECA

$\begin{array}{lll}\text { Alemania } & 0.78(0.64-0.95)^{*} & 0.72(0.60-0.88) \\ \text { Dinamarca } & 0.78(0.36-1.70) & 0.91(0.44-1.90) \\ \text { Francia } & 0.97(0.16-5.83) & 1.60(0.29-8.81) \\ \text { Reino Unido } & 0.71(0.39-1.30) & 0.67(0.38-1.21) \\ \text { Suecia } & 0.61(0.23-1.63) & 0.55(0.21-1.45)\end{array}$

\section{ECV}

FC $0.72(0.64-0.81)^{*}$ $1.03(0.59-1.77)$ $0.42(0.16-1.12)$ $0.02(0.00-0.12)$ $0.77(0.45-1.34)$ $0.15(0.02-1.08)$
$0.49(0.28-0.85)$

$0.51(0.27-0.95)$

$0.61(0.15-2.42)$
Comp

$* \mathrm{p}<0.05 . \mathrm{IAM}=$ infarto agudo de miocardio, $\mathrm{ECA}=$ evento coronario agudo, $\mathrm{ECV}=$ evento vascular cerebral, $\mathrm{FC}=$ falla cardiaca, $\mathrm{Comp}=\mathrm{eventos}$ cardiovasculares compuestos 
La información respecto del riesgo cardiovascular que se extrae de esta base, datos de vida real, de 5 países en Europa es concordante con la reflejada en estudios primarios que han evaluado la asociación de riesgo cardiovascular con uso de vildagliptina y permite identificar un perfil de riesgo cardiovascular no incrementado para este DPP4-I.

Bekiari 2016 (32) - Revisión sistemática de ECC (69 estudios 28006 pacientes) en eficacia y seguridad vildagliptina versus placebo u otro hipoglucemiante oral. En cuanto a los desenlaces de seguridad esta RS incluyo estudios que evaluaron eventos cardio- cerebro vascular, además de muerte de causa vascular.
De manera global, vildagliptina comparada con placebo demostró reducción (diferencia media ponderada), de niveles de HbA1c en $0.69 \%$; (IC 95\% 0.56-0.83).

La efectividad comparativa con el conjunto de otros hipoglucemiantes: metformina, sulfonilurea, $\alpha$ glucosidasa inhibidor, sitagliptina, pioglitazona, incluyendo insulina fue de $0.01 \%$; (IC95\% 0.16-0.14).

Los resultados de efectividad que representan, al menos, el 70\% ponderado de las comparaciones y los desenlaces de seguridad cardiovascular sintetizados en la tabla 6 Bekiari 2016.

Tabla 7 Bekiari 2016 - Efectividad y desenlaces cardiovasculares

\section{Vildagliptina versus placebo o comparador activo antidiabético oral}

\begin{tabular}{|c|c|c|c|}
\hline Efectividad & Placebo & Metformina (19\%) & Sulfonilurea (48\%) \\
\hline Reducción HbA1c% & $-0.69(-0.83--056)$ & $0.14(-0.38-0.66)$ & $-0.05(-0.21-0.11)$ \\
\hline Posibilidad de lograr HbA1c $<7 \%$ - & & OR & \\
\hline Vildagliptina sola & & $2.76(1.27-6.00)$ & \\
\hline Vildagliptina más metformina & & $2.67(2.06-3.46)$ & \\
\hline $\begin{array}{l}\text { Vildagliptina más metformina } \\
\text { versus comparador activo }\end{array}$ & & $0.89(0.78-1.02)$ & \\
\hline Seguridad & & OR & \\
\hline Incidencia de falla cardíaca & & $0.77(0.46-1.30)$ & \\
\hline Evento cardio-cerebro vascular & & $0.91(0.73-1.14)$ & \\
\hline Muerte de causa cardiovascular & & $1.23(0.69-2.19)$ & \\
\hline
\end{tabular}

Filion 2016 (33) - En la misma línea de los estudios observacionales, pero bajo diseño de caso control anidado, con alto poder representado en una cohorte integrada (1'499.650 pacientes) y tiempo de observación equivalente a 3 millones de personas año en Canadá, Estados Unidos y el Reino Unido, el grupo CNODES (Canadian Network for Observational Drug Effect Studies), buscó responder a la pregunta de si el uso de las incretinas (como grupo) comparadas con otros hipoglucemiantes orales en el tratamiento de DBT-2 incrementa riesgo de eventos cardiovascular no deseados, específicamente falla cardíaca.

La población - mayores de 18 años - en esta cohorte la integraron pacientes diabéticos que hubiesen recibido; además de
DPP-4I, otros antidiabéticos orales (biguanidas, sulfonilureas, tiazolidindionas, análogos GLP-1, alfa-glucosidasas inhibidores, meglitinidas, inhibidores de cotransportadores sodio-glucosa; solos o en combinación). Dos grupos fueron diferenciados dentro de la cohorte: pacientes con y sin falla cardíaca identificada (registro clínico) con anterioridad o en el mismo día de ingreso a la cohorte.

La tasa cruda - incidencia - fue de 9.2 eventos 1000 personas año para la totalidad de la cohorte. Ahora bien, para el grupo con falla cardíaca identificada previamente la incidencia fue de 43.5 eventos 1000 personas año y para el grupo sin falla previamente identificada fue 7.5 eventos 1000 personas año. 
Al realizar el análisis para el grupo de pacientes sin historia de falla cardíaca y bajo tratamiento con incretina, comparando por quienes recibieron otro antidiabético, no hubo riesgo aumentado para admisión por insuficiencia cardíaca HR 0.82; (IC95\% 0.671.00) Cuando se compara entre incretinas: DPP-4I versus análogos GLP-1, no hay diferencias en el riesgo HR 0.84 (IC95\% 0.69 - 1.02) y HR 0.95 (IC95\% 0.83 - 1.10) respectivamente.

De otra parte el riesgo de hospitalización por falla en los pacientes con historia de falla cardíaca y bajo tratamiento con incretina, comparando por quienes recibieron otro antidiabético no fue mayor: HR 0.86; (IC95\% 0.62 - 1.19). Tampoco se identificaron diferencias entre DPP-4I versus análogos GLP-1 HR 0.87 (IC95\% 0.63-1.21) y HR 0.75 (IC95\% 0.22-2.51).

Kongwatcharapong 2016 (34) Revisión sistemática y me- ta-análisis de 54 ECCAs (74737 pacientes), para evaluar ocurrencia de falla cardíaca en pacientes bajo tratamiento con DPP4-I, ampliando los períodos de observación a con estudios que superaran 12 semanas y que además incluyeran características criticas al riesgo cardiovascular/falla cardiaca, no contemplados en estudios previos.

La composición de los 54 ECCAs por cada DPP4- I fue la siguiente: 9 en alogliptina 7 en linagliptina 9 en saxagliptina, 19 en sitagliptina y 10 en vildagliptina, entre estos últimos 6 con comparador placebo y 4 con comparador activo (glimepirida, rosiglitazona, metformina, gliclazida)

En la tabla 8 Kongwatcharapong 2016 están agrupados los riesgos para falla cardíaca para los diferentes DPP4-I y en función del riesgo cardiovascular.

\begin{tabular}{|c|c|c|c|}
\hline \multicolumn{4}{|c|}{ Riesgo de falla cardíaca por tipo de DPP-4I y riesgo cardiovascular $R R($ IC $95 \% 9$ ) } \\
\hline & Todos los estudios & Bajo riesgo CV & Alto riesgo CV \\
\hline Alogliptina & $1.17(0.90-1.52)$ & $1.11(0.50-2.44)$ & $1.18(0.89-1.55)$ \\
\hline Linagliptina & - & $1.16(0.59-2.29)$ & - \\
\hline Saxagliptina & $1.21(1.02-1.43)$ & $0.53(0.23-1.24)$ & $0.02(0.00-0.12)$ \\
\hline Sitagliptina & $0.71(0.39-1.30)$ & $0.67(0.38-1.21)$ & $1.25(1.06-1.49)$ \\
\hline Vildagliptina & - & $0.81(0.50-1.31)$ & - \\
\hline
\end{tabular}

Con base en los RR identificados en este meta-análisis se observa incremento significativo de $25 \%$ en el riesgo para falla cardíaca, específicamente en pacientes con alto riesgo cardiovascular, para uno de los DPP -4I, saxagliptina.

Sato 2016 (35) - Estudio observacional con 962 pacientes hospitalizados por falla cardíaca $293(30.5 \%)$ de ellos con DBT 2 y en tratamiento con DPP-4 I 122 pacientes, dentro de los que 44 (36\%) recibían vildagliptina. El objetivo del estudio buscó identificar la asociación entre tratamiento con DPP-4 I con muerte por causa cardiovascular y por toda causa emparejando grupos con y sin DPP-4 I, y análisis para lograr puntaje de propensión.

El análisis para muerte cardiovascular identificó 4.8\% pacientes con DPP-4I versus $18,1 \%$ pacientes sin DPP-4I; p 0.015 y en muerte por todas las causas $14.5 \%$ versus $41 \%$ respectivamente p 0.003 .
El análisis de riesgos proporcionales, ajustado por variables confusoras, identifico a los DPP-4I como factor predictor independiente para mortalidad por toda cusa HR $0.46, \mathrm{p} 0.010$ en cohorte antes de emparejamiento y HR 0.37 p 0.003 luego de emparejar para pacientes en quienes concurre falla cardiaca y DBT.

McInnes 2015 (36) - Meta-análisis de 40 ECCAs fase III-IV, incluyó 17000 pacientes en quienes se exploraron y analizaron los aspectos de seguridad cardiovascular y falla cardíaca atribuible vildagliptina. Los estudios primarios seleccionados reclutaron pacientes con alto riesgo cardiovascular, además de diabetes bajo tratamiento, tenían insuficiencia cardíaca identificada y compromiso renal moderado/severo.

Fueron identificados 9599 sujetos que recibían vildagliptina (exposición calculada a vildagliptina: 9251 personas-año), 7847 sujetos que recibían medicamento diferente a vildagliptina (exposición calculada: 7317 personas-año). 
El comparador fue en el $36 \%$ placebo, sulfonilurea 33\%, metformina 15\%, tiazolidindionas $10 \%$ y $6 \%$ otros antidiabéticos orales. Vale mencionar que en cada grupo analizado, vildagliptina (9.7\%) y comparador (11.0\%) se trataba de población hispana o latina.

El evento cardiovascular adverso mayor (MACE) registrado en el grupo de vildagliptina fue para 83 pacientes $(0.86 \%)$ y en 85 de quienes recibían otra terapia (1,2\%); HR 0.82 (IC95\% 0.61-1.11). En la tabla 9 McInnes 2015 se sintetizan los desenlaces cardiovasculares comparados.

Tabla 9. McInnes 2015 Desenlace cardiovascular vildagliptina versus otro antidiabético oral

\begin{tabular}{llcc}
\hline \multicolumn{4}{c}{ Vildagliptina versus otro Antidiabético oral Incidencia (IC 95\%) } \\
\hline $\begin{array}{l}\text { Vildagliptina } \\
\text { n/N }(\%)\end{array}$ & $\begin{array}{c}\text { Comparador activo } \\
\mathrm{n} / \mathrm{N}(\%)\end{array}$ & $\begin{array}{c}\text { RR M-H } \\
(\text { IC } 95 \%)\end{array}$ \\
\hline MACE & $83 / 9599(0.86)$ & $85 / 7102(1.20)$ & $0.82(0.61-1.11)$ \\
Infarto miocardio & $38 / 9599(0.40)$ & $35 / 7102(0.49)$ & $0.87(0.56-1.38)$ \\
Evento Cerebro-Vascular & $24 / 9599(0.25)$ & $25 / 7102(0.35)$ & $0.84(0.47-1.50)$ \\
Muerte cardio-vascular & $25 / 9599(0.26)$ & $28 / 7102(0.39)$ & $0.77(0.45-1.31)$
\end{tabular}

RR: riesgo relativo, M-H: Mantel-Haenszel, MACE: Major Adverse Cardio Vascular Events, IC: intervalo de confianza

\section{Vildagliptina en compromiso renal}

El razonamiento clínico y juicio crítico que demanda el cuidado de los pacientes con DBT obliga a proceder sin desestimar las implicaciones por el riesgo y compromiso en la función renal que, a su vez, impone retos en la selección y uso diferencial de las alternativas farmacológicas.

La presencia y progresión de albuminuria de en personas con DBT se ha identificado como un fuerte indicador de incremento para mortalidad cardiovascular; 8 veces en relación con la población general y entre 2 y 4 veces en comparación con diabéticos sin desarrollo de proteinuria $(37,38)$. A su vez, el descenso en la tasa de filtración glomerular junto con presencia de albuminuria son factores de riesgo independientes para eventos cardiovasculares (39).

Ahora bien, las implicaciones por eventos hipoglucémicos en diabéticos con función renal deteriorada son todavía más demandantes en la medida en que las variaciones hipoglucémicas representan mayor probabilidad de riesgo fatal (40), particularmente cuando se plantea intensificación de tratamiento pretendiendo lograr niveles "normales" de HbA1c. El estudio ACCORD - Action to Control Cardiovascular Risk in Diabetes -para evaluar respuesta fue detenido en forma temprana al identificar mayor mortalidad en el grupo de intensificación de terapia, atribuido a incidencia mayor de eventos hipoglucémicos $(41,42)$.

Es así que, en el contexto de DBT con función renal comprometida, las propiedades pleiotrópicas y efectividad de los DPP-4I para el control metabólico son una clase de medicamentos con ventaja particularmente deseable al reducir el riesgo de hipoglucemia.
Howse 2016 (43) - Revisión sistemática y meta-análisis de 13 ECCAs -6848 pacientes diabéticos con función renal comprometida, para evaluar cambio en $\mathrm{HbAlc}$, hipoglucemia, eventos cardiovasculares, muerte y enfermedad renal terminal asociadas a tratamiento con incretinas. Once de los estudios fueron realizados para evaluar DPP-4I.

De manera global, comparada con placebo, la clase incretinas demostró reducción significativa en los niveles de $\mathrm{HbA} 1 \mathrm{c}$ con diferencial media 0.64; (IC95\% 0.79-0.48); sin diferencias significativas con comparadores activos: media pondera 0.7 ; (IC95\% $0.25-0.12)$.

En general, el riesgo de hipoglucemia comparado con placebo identificó RR 1.38; IC95\% 1.01-1.89), sin diferencias con comparadores activos RR 0.24; (IC95\% 0.03-1.94).

En mortalidad las incretinas versus placebo reflejaron RR 1.21; (IC95\% 0.64-2.29), con comparador activo RR, 0.70; (IC95\% $0.32-1.54)$.

Es interesante observar, para el desenlace meta-analítico riesgo de hipoglucemia RR 1.38; (IC95\% 1.01-1.89), la manera en que se distribuye el riesgo en los diferentes estudios. El estudio de tolerabilidad y eficacia de vildagliptina en pacientes con DMT2 e insuficiencia renal crónica moderada o severa, tiempo de observación a 52 semanas, análisis en 369 sujetos, (Kothny 2012), exhibe un riesgo puntual 0.35; (IC95\% 0.09-1.42), tendencia a la reducción del riesgo, en contraste con lo identificado para las demás incretinas. Este desenlace fue destacado de manera semejante en la revisión publicada por Thomas y cols (36). 
Con base en los datos disponibles en los archivos suplementarios de ésta revisión calculamos el riesgo de muerte por toda causa para cada uno de los DPP-4I versus placebo, para análisis, disponible en la tabla 10 House 2016.

Tabla House 102016 - Riesgo de muerte por toda causa -DDP-4I versus Placebo

\begin{tabular}{|c|c|c|c|}
\hline \multicolumn{4}{|c|}{ Riesgo de muerte por toda causa en DBT función renal comprometida } \\
\hline & $\begin{array}{l}\text { DPP-4I } \\
\text { n/N }\end{array}$ & $\begin{array}{c}\text { Placebo } \\
\text { n/N }\end{array}$ & RR (IC 95\%) \\
\hline Linagliptina (Laakso 2013) & $1 / 113$ & $1 / 122$ & $1.07(0.06-17.13)$ \\
\hline Linagliptina (McGill 2013) & $3 / 68$ & $3 / 65$ & $0.95(0.19-4.60)$ \\
\hline Sitagliptina (Chan 2018) & $5 / 65$ & $1 / 26$ & $1.92(0.24-15.03)$ \\
\hline Vildagliptina (Kothny 2012) & $8 / 287$ & $6 / 226$ & $1.04(0.36-2.98)$ \\
\hline
\end{tabular}

Thomas 2016 - (44) Revisión sistemática con siete ensayos clínicos que evaluaron reducción de HbAlc, glucosa en ayunas, hipoglucemia y tolerabilidad en pacientes que cursaban con insuficiencia renal asociada.

Con base en los estudios incluidos en esta revisión fueron identificados dos DPP-4I que soportan eficacia en disminución de $\mathrm{HbAlc}$ en pacientes con diferente grado de severidad respecto a la insuficiencia renal y tiempo de observación hasta la semana 52 de tratamiento.

En forma global, con independencia el grado de compromiso renal, con vildagliptina la reducción promedio de $\mathrm{HbA} 1 \mathrm{c}$ fue $0,8 \%$, desde una línea de base de $7,8 \%$ en semana 12 que se mantiene hasta la semana 52.

En pacientes con insuficiencia renal moderada la reducción en el grupo vildagliptina fue de $0,7 \%$ en las semanas 12 y 52 y en pacientes con insuficiencia renal severa la reducción alcanzada fue $0,8 \%$.

Kothny 2015 (45) - Desde el ECCA de 2012 con 369 pacientes con DMT2 e insuficiencia renal crónica (IRC) moderada o severa, tiempo de observación a 52 semanas para evaluar seguridad y eficacia de vildagliptina (50 mg/1 dosis/día) añadido a la terapia antidiabética, que en su mayoría tenían de base tratamiento insulina, demostró un cambio medio ajustado en $\mathrm{HbA1C}$ en $-0,4 \pm$ $0,1 \%$ en pacientes con IR moderada y $-0,7 \pm 0,2 \%$ en pacientes con IR severa y un perfil de seguridad similar a placebo.

Para 2015 Kothny reporta la evaluación vildagliptina comparada con sitagliptina en diabéticos con función renal comprometida identificando reducciones de HbAlc semejantes -0.54 y $-0.56(\mathrm{p}=0.87)$. El porcentaje de pacientes que lograron meta de
HbAlc $\leq 7.0 \%$ fue similar en ambos grupos, merece destacar que para $\mathrm{HbA} 1 \mathrm{c} \leq 6.5 \%$ el porcentaje fue significativamente mayor (el doble) en el brazo $29.0 \%$ versus $14.3 \%$ en sitagliptina.

\section{Vildagliptina en Insulinoterapia}

Debido a la pérdida progresiva de la función de la célula $\beta$ una proporción variable de pacientes requerirán insulina; con tolerabilidad y control metabólico en unos $(46,47)$, pero en otros el resultado es pobre y; aún bajo esquemas de intensificación, los desenlaces en de reducción de riesgo o progresión de daño cardiovascular y mortalidad no se logran para cerca de las tres cuartas partes de quienes se encuentran bajo combinación insulina más antidiabético oral $(48,49)$.

Adicionalmente los riesgos de hipoglucemia complican el espectro clínico (50) dificultad compleja de sortear a la hora de acordar con el paciente el plan de cuidado a seguir.

De manera progresiva hay nueva información sobre el efecto en el control metabólico en pacientes diabéticos con la asociación gliptina más insulina. En éste sentido y específicamente en lo referente a vildagliptina a continuación exponemos evidencia reciente disponible.

Kanazawa 2017 (51) -ECCA abierto en siete centros de Japón en diabéticos bajo insulinoterapia (larga acción, corta, premezclada), asignados en forma aleatoria a recibir vildagliptina $50 \mathrm{o}$ 100 miligramos día según determinación y criterio de médico tratante, o no recibir vildagliptina. Los ajustes en dosis de insulina fueron realizados según criterio médico, el diseño especificó no ajustes en el DPP-4I. 
Los desenlaces a evaluar fueron el cambio en HbA1c y eventos hipoglucémicos, a dos años de observación bajo tratamiento.

Los niveles de HbAlc se redujeron de manera significativa en los pacientes tratados con vildagliptina en $-0.58 \pm 0.16 \%$ en comparación con el grupo control $0.19 \pm 0.19 \%$; p 0.005. Fue evidente y de manera significativa el efecto en el cambio en $-0.63 \pm$ $0.17 \%$ de $\mathrm{HbAlc}$ atribuible a la dosis 100 miligramos día de vildagliptina en comparación con quienes recibieron 50 miligramos día $-0.38 \pm 0.38 \%$.

Al final del período de observación (52 semanas) el 40.5\% de los pacientes que recibieron vildagliptina lograron niveles $\mathrm{HbA} 1 \mathrm{c}$ $<7 \%$ y el $70.3 \% \mathrm{HbA} 1 \mathrm{c}<8 \%$, en tanto que para el grupo con- trol la proporción de pacientes que lograron esos niveles fue de $19.4 \%$ y $41.7 \%$ respectivamente.

El análisis incluyó explorar la asociación entre las características basales de los pacientes y el efecto atribuible a vildagliptina. El coeficiente de correlación (r) para el cambio en HbA1c en el análisis no ajustado fue directo y significativo con los niveles de creatinina e inverso y significativo con el nivel de partida de $\mathrm{HbA1c}$. Estas correlaciones se mantuvieron en el mismo sentido y también significativas al realizar el ajuste por edad (Modelo 1), así como al ajustar en forma simultanea por edad, tiempo de duración de la diabetes y el índice de masa corporal (Modelo 2). Ver tablas 11 y 12 Kanazawa 2017 a) y b).

Tabla I I - Kanazawa 2017 a- Cambio HbA1c

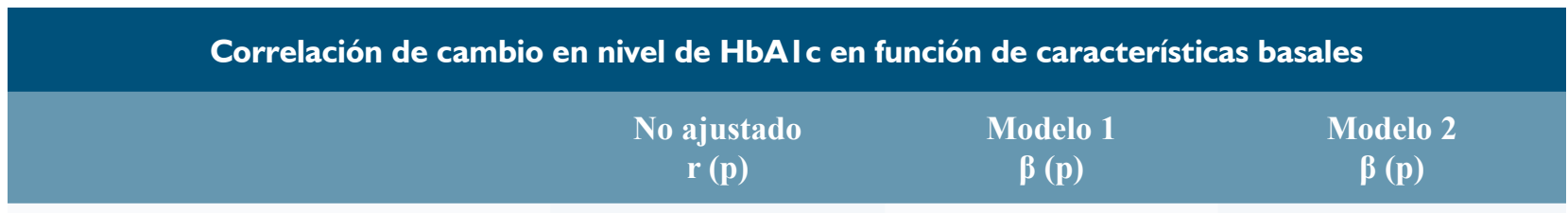

Características de base

\begin{tabular}{|c|c|c|c|}
\hline -Edad & $0.11(0.53)$ & - & - \\
\hline -Duración de la DBT & $0.07(0.69)$ & $0.00(0.99)$ & - \\
\hline -Índice de masa corporal & $0.05(0.78)$ & $0.10(0.59)$ & - \\
\hline -Creatinina sérica & $0.42(0.016)$ & $0.47(0.016)$ & $0.48(0.03)$ \\
\hline -Filtración glomerular estimada & $-0.32(0.07)$ & $-0.42(0.06)$ & $-0.38(0.16)$ \\
\hline -HbAlc & $-0.39(0.01)$ & $-0.40(0.02)$ & $-0.38(0.04)$ \\
\hline -Dosis día de insulina & $-0.08(0.66)$ & $-0.03(0.89)$ & $-0.03(0.88)$ \\
\hline
\end{tabular}

De otra parte, en cuanto a eventos hipoglucémicos identificados en los grupos, hay clara disminución de los mismos en el grupo que recibió vildagliptina y la manera en que los episodios (OR) por año caen clínica y estadísticamente significativa (desde tres o más episodios) para el grupo de pacientes en quienes se adiciona vildagliptina a insulinoterapia. 
Riesgo de hipoglucemia en adición de vildagliptina a insulinoterapia

Vildagliptina $\quad$ Control OR (IC95\%)

Episodio hipoglucemia por año

-Uno o más

-Dos o más

-Tres o más

-Cuatro o más
14

10

4

1
19

13

11

9
$0.55(0.21-1.38)$

$0.66(0.24-1.77)$

$0.28(0.08-0.97)$

$0.55(0.01-0.70)$

OR: odss ratio, IC: intervalo de confianza

Merece resaltar la disminución promedio de las dosis totales diarias de insulina en -5.8 unidades; en carga -4.0 unidades y basales -1.6 unidades para el grupo que recibió vildagliptina en comparación con lo propio en el grupo control $+0.2 ;-0.2 ;+0.4$ unidades respectivamente.

Li 2017 (52) - ECC doble enmascarado a 24 semanas en adultos con edad promedio 59 años, DBT 2 de más de 10 años de evolución - no controlada con HbA1c en media de 8,6\% - aún bajo tratamiento con insulina o insulina + metformina. Se comparó el efecto sobre HbA1c y para variaciones glucémicas; mediante el cambio basal-final de la media de la amplitud de la excursión glucémica - (MAGE), particularmente nocturna (00:00 a 00:06 horas), al adicionar vildagliptina.

El cambio final en los niveles HbA1c para el grupo en insulina + metformina+vildagliptina fue significativo de $8.5 \pm 0.8 \%$ a. 7.6 $\pm 0.8 \%, \mathrm{p}<0.01$, en el grupo insulina + metformina + placebo fue de $8.8 \pm 0.7 \%$ a $8.9 \pm 1.3 \%, \mathrm{p}>0.05$.

La variación glucémica - MAGE - en el grupo que recibió vildagliptina fue significativamente menor $3.7 \pm 0.8 \mathrm{mmol} / 1$ vs 5.3 $\pm 2.6 \mathrm{mmol} / \mathrm{l}$, p 0.04 .

Gautier 2016 (53) Estudio piloto, ECC abierto incluyó 38 pacientes aleatorizados en dos grupos para comparar y analizar, por intención a tratar, dos esquemas terapéuticos en pacientes diabéticos con antidiabético oral y en quienes por pobre control se asociaría insulina. Un grupo mantendría el esquema previo (metformina + sulfonilurea) y se adicionó insulina, para el otro recibiría (metformina + vildagliptina) con adición insulina.

Los desenlaces a evaluar: control glucémico HbA1c $<7.0 \%$ e incidencia de hipoglucemia a 12 y 24 semanas.

De manera global y significativamente a las 12 semanas hubo mayor proporción de pacientes en el grupo al que se adicionó vildagliptina con el logro de HbA1c $<7.0 \%$ : 47.4\% vs $11.8 \%$, p 0.021 , sin incremento del riesgo de hipoglucemia. Hacia el final del período de observación - 24 semanas - la proporción de pacientes en quienes de identificó hipoglucemia fue numéricamente diferente $44.4 \%$ vs. $47.1 \%$, p 0.877 .

Chen 2015 (55) - Revisión sistemática y meta-análisis de siete ECC - reunió 3384 pacientes para analizar efectividad y seguridad de DPP-4I en combinación con insulina en adultos con DBT2, Ente ellos fue incluido el estudio de Fonseca 2007 con 296 pacientes cuya tiempo medio de enfermedad era de 15 años y en insulinoterapia basal o mixta.

El meta-análisis de los siete estudios (todos los DPP-4I) revelo reducción de $\mathrm{HbA1c}$ en -0.52; (IC95\% 0.44-0.59), en el que el resultado para vildagliptina (a 24 semanas de seguimiento) fue -0.30; (IC95\% 0.02-0.58) en comparación con placebo o comparador activo hipoglucemiante oral. No hubo incremento significativo en el riesgo de hipoglucemia ni en el agregado del meta-análisis 1.04; (IC95\% 0.832-1.31), ni para vildagliptina en forma aislada 0.77 ; (IC95 \% 0.53-1.14)

Kim 2016 (54) - Revisión sistemática y meta-análisis con 9 ECCAs (4464 adultos DBT 2) para análisis comparativo de desenlaces de efectividad (cambio en HbA1c) y seguridad (hipoglucemia) entre esquema DPP-4I + Insulina versus Insulina + placebo.

En la RS fueron incluidos dos estudios con vildagliptina. Uno de ellos (Fonseca 2007) bajo esquema vildagliptina + insulina versus insulina + placebo y el otro (Kothny 2013) bajo esquema vildagliptina + metformina + insulina versus insulina + placebo + metformina, ambos estudios a 24 semanas y cada uno aportó el 10\% del peso ponderado del tamaño muestra para el meta-análisis. En la tabla $13 \mathrm{Kim} 2016$, cambio en HbA1c para cada uno de los 9 estudios, riesgo para evento hipoglucémico y correspondiente indicador meta-analítico. 


\section{Cambio en nivel de HbAlc}

DPP-4I + Ins

Ins + plb

ECCA

media (DE)

media (DE)

media (IC 95\%)

RR (IC95\%)

Alogliptina

-Rosenstock 2009

-Kaku 2014

$\begin{array}{ll}-0.71(0.08) & -0.13(0.08) \\ -0.96(0.06) & -0.29(0.06)\end{array}$

$-0.59(-080--0.38)$

$1.14(0.75-1.73)$

$-0.66(-0.82--0.50)$

$0.99(0.57-1.71)$

Linagliptina

-Yld-Jarvinen 2013

$-0.58(0.08)$

$-0.65(-0.87--043)$

$0.95(0.77-1.17)$

Saxagliptina

-Barnett 2012

$-0.73(0.05)$

$-0.07(0.08)$

$-0.41(-0.59--0.23)$

$0.93(0.62-1.38)$

Sitagliptina

-Kadowaki 2013

-NCT01462266

-Vilsbøll 2010

Vildagliptina

-Kothny 2013

-Fonseca 2007

$\begin{array}{ll}-0.60(0.10) & -0.30(0.10) \\ -1.31(0.06) & -0.87(0.06) \\ -0.59(0.06) & -0.03(0.06)\end{array}$

$-0.32(0.07)$

$.03(0.06)$

$1.62(0.93-2.85)$

$0.65(0.52-0.85)$

$$
\begin{aligned}
& -0.90(-1.05--0.75) \\
& -0.45(-0.61--0.29) \\
& -0.56(-0.70--0.42)
\end{aligned}
$$

$-0.10(0.10)$

$-0.20(0.10)$

$$
-0.30(-0.50--0.10)
$$

Meta-análisis

(Heterogeneidad I ${ }^{2}$ 76.4\%)

$-0.58(-0.70--0.46)$

$0.94(0.84-1.05)$

DPP-4I: dipeptidil peptidasa 4 inhibidor, HbA1C: hemoglobina glucosilada, RR: riesgo relativo, DE: desviación estándar, IC: intervalo de confianza.

Chen 2015 (55) - Revisión sistemática y meta-análisis de siete ECC - reunió 3384 pacientes para analizar efectividad y seguridad de DPP-4I en combinación con insulina en adultos con DBT2, Ente ellos fue incluido el estudio de Fonseca 2007 con 296 pacientes cuya tiempo medio de enfermedad era de 15 años y en insulinoterapia basal o mixta.

El meta-análisis de los siete estudios (todos los DPP-4I) revelo reducción de HbA1c en -0.52; (IC95\% 0.44-0.59), en el que el resultado para vildagliptina (a 24 semanas de seguimiento) fue -0.30; (IC95\% 0.02-0.58) en comparación con placebo o comparador activo hipoglucemiante oral.

No hubo incremento significativo en el riesgo de hipoglucemia ni en el agregado del meta-análisis 1.04; (IC95\% 0.832-1.31), ni para vildagliptina en forma aislada 0.77 ; (IC95 \% 0.53-1.14). 
Ito 2015 (56) Estudio abierto prospectivo, multicéntrico -Japón- en 57 pacientes con DBT bajo insulinoterapia con pobre control (con $\mathrm{HbAlc}>8 \%$ ), al menos 10 años bajo tratamiento, para quienes se propuso determinar, en 36 de ellos, la eficacia a 24 semanas por la adición de 100 miligramos día de vildagliptina en combinación con insulina registrando valor promedio logrado en HbA1c de 7.5\%, de manera notable las dosis de insulina fueron reducidas en 8,3 unidades promedio.

Para los 21 pacientes restantes se propuso comparar la combinación sitagliptina-insulina 12 semanas versus vildagliptina + insulina 12 semanas con cambio de HbAlc en $0.7 \%$, de $9.0 \%$ a $8.3 \%$ con mínimos o ningún ajuste en las dosis media de insulina.

\section{Discusión}

El espectro de resultados expuestos hace parte de una continuada investigación en el DPP-4I vildagliptina; en 2018 supera 10 años de investigaciones previas, y adquiere especial interés en pacientes de edad avanzada, con diabetes de larga data y en quienes el riesgo de eventos hipoglucémicos, eventos cardiovasculares y deterioro de función renal es particularmente alto.

De manera global, el conjunto de publicaciones revisadas para el DPP-4I vildagliptina; sola o en combinación con metformina, también cuando se la adiciona tempranamente a pacientes bajo terapia insulínica, refleja perfil de efectividad y seguridad altamente confiables; semejante en frecuencia de eventos no deseados a placebo. En comparación con hipoglucemiantes orales más frecuentemente utilizados, destaca el menor riesgo de hipoglucemia y efecto neutro para ganancia de peso.

En esta revisión y síntesis de evidencias resultantes del conjunto de 23 estudios publicados entre 2015 a 2018 permite analizar el perfil de beneficios en efectividad y seguridad del DPP-4I vildagliptina en pacientes con DM2, particularmente para quienes de manera manifiesta o latente integran; cuando menos, aquella mitad de diabéticos con algún grado de compromiso crónico y progresivo en la función renal (57), hecho que además de incrementar el riesgo de eventos cardiovasculares fatales y no fatales (58), impone restricciones; en diverso grado e incluso contraindicación para el uso de metformina y de otro tipo de hipoglucemiantes de larga acción.

Entre los más recientes y robustos estudios con los que se identifica no deterioro o no incremento de eventos vasculares o no riesgo incrementado de muerte de origen cardiovascular en DM2 bajo tratamiento con vildagliptina hay que destacar la RS de Bekiari y cols (32) y el estudio de base poblacional en 5 países de Europa-Williams 2017 (31).

Suma también el estudio para evaluar el efecto de vildagliptina sobre la función ventricular en pacientes con diabetes y fracción de eyección reducida - VIVIDD - en el que la dosis de 50 miligramos dos veces al día, comparada con placebo, no reflejo impacto negativo (27). Naturalmente deben continuar las evaluaciones para esclarecer las discrepancias reportadas en el grupo de incretinas; incluidas las gliptinas, por el efecto sobre la función cardíaca, insuficiencia cardíaca y riesgo de admisión hospitalaria (59).

Entre tanto, es necesario reconocer el importante giro en la concepción de la DM2 como enfermedad y el renovado enfoque terapéutico actual, en buena parte impulsado por la investigación de los DPP-4I. Es así que nos alejamos del abordaje predominantemente centrado en la "meta HbAlc ideal" para evolucionar hacia una mejor comprensión de la alteración endotelial sistémica y desorden vascular que subyace en la fisiopatología de la diabetes mellitus, una de las condiciones crónicas más comunes en la población mundial, que genera serios y difíciles retos al paciente a su familia y que trascienden a los sistemas de salud y en la economía global.

\section{Referencias}

1. Nichols GA, Gullion CM, Koro CE, Ephross SA, Brown JB. The incidence of congestive heart failure in type 2 diabetes: an update. Diabetes care. Am Diabetes Assoc; 2004; 27(8):1879-84.

2. Tonelli M, Muntner P, Lloyd A, et al. Risk of coronary events in people with chronic kidney disease compared with those with diabetes: a population-level cohort study. Lancet. 2012; 380(9844):807-14.

3. Shurraw S, Hemmelgarn B, Lin M, et al. Association between glycemic control and adverse outcomes in people with diabetes mellitus and chronic kidney disease: a population-based cohort study. Arch Intern Med. 2011; 171(21):1920-7.

4. Röhrborn D, Wronkowitz N, Eckel J. DPP4 in Diabetes. Front Immunol. 2015 Jan 4; 6:386.

5. Scherbaum, Schweizer, Mari, Nilsson, Lalanne, Wang, et al. Evidence that vildagliptin attenuates deterioration of glycaemic control during 2-year treatment of patients with type 2 diabetes and mild hyperglycaemia*. Diabetes, Obesity and Metabolism. 2008;10(11):1114-24.
6. Schweizer A, Dejager S, Bosi E. Comparison of vildagliptin and metformin monotherapy in elderly patients withtype 2 diabetes: a 24-week, double-blind, randomized trial. Diabetes Obes Metab 2009 Aug;11(8):804-12.

7. Matthews D, Dejager S, Ahren B, Fonseca V, Ferrannini E, Couturier A, Foley JE, Zinman B.. Vildagliptin add-on to metformin produces similar efficacy and reduced hypoglycaemic risk compared with glimepiride, with no weight gain: results from a 2-year study. Diabetes Obes Metab. 2010 Sep;12(9):780-9.

8. Strain W, Lukashevich V, Kothny W, Hoellinger MJ, Paldánius PM. Individualised treatment targets for elderly patients with type 2 diabetes using vildagliptin add-on or lone therapy (INTERVAL): a 24 week, randomised, double-blind, placebo-controlled study.. Lancet 2013 Aug 3;382(9890):409-16.

9. Kothny W, Foley J, Kozlovski P, Shao Q, Gallwitz B, Lukashevich V. Improved glycaemic control with vildagliptin added to insulin, with or without metformin, in patients with type 2 diabetes mellitus. Diabetes Obes Metab. 2013 Mar;15(3):252-7. 
10. Lukashevich V, Prato SD, Araga M, Kothny W. Efficacy and safety of vildagliptin in patients with type 2 diabetes mellitus inadequately controlled with dual combination of metformin and sulphonylurea. Diabetes Obes Metab. 2014 May;16(5):403-9.

11. Derosa G, Bonaventura A, Bianchi L, Romano D, Fogari E, D'Angelo A, Maffioli P. Vildagliptin compared to glimepiride on post-prandial lipemia and on insulin resistance in type 2 diabetic patients. Metabolism. 2014 jul;63(7):957-67.

12. Garber AJ, Abrahamson MJ, Barzilay JI, et al. Consensus statement by the American Association of Clinical Endocrinologists and American College of Endocrinology on the comprehensive type 2 diabetes management algorithm-2017. Executive summary. Endocr Pract. 2017; 23:207-238.

13. Khangura S, Konnyu K, Cushman R, Grimshaw J, Moher D. Evidence summaries: the evolution of a rapid review approach. Syst Rev. 2012 Feb $5 ; 1: 10$.

14. Polisena J, Garritty C, Kamel C, Stevens A, Abou-Setta A. Rapid review programs to support health care and policy decision making: a descriptive analysis of processes and methods. Systematic Reviews. 2015; 4(1).

15. Shea BJ, Reeves BC, Wells G, Thuku M, Hamel C, Moran J, Moher D, Tugwell P, Welch V, Kristjansson E, Henry DA. AMSTAR 2: a critical appraisal tool for systematic reviews that include randomised or non-randomised studies of healthcare interventions, or both. BMJ. 2017 Sep 21;358:j4008.

16. Downs, Black. The feasibility of creating a checklist for the assessment of the methodological quality both of randomised and non-randomised studies of health care interventions. Journal of Epidemiology and Community Health. 1998;52(6):377-84.

17. Cohen C, Davis C, Shalev V, Chodick G. Effectiveness of vildagliptin as add-on to metformin monotherapy among uncontrolled type 2 diabetes mellitus patients in a real-world setting. J Diabetes. wiley; 2018;10(1):68-72.

18. Ahrén B, Simonsson E, Larsson H, Landin-Olsson M, Torgeirsson H, Jansson P-AA, et al. Inhibition of dipeptidyl peptidase IV improves metabolic control over a 4-week study period in type 2 diabetes. Diabetes Care. 2002 May 3;25(5):869-75.

19. Wang L-G, Wang H, Liu Q, Hua W-C, Li C-M. A network meta-analysis for efficacy and safety of seven regimens in the treatment of type II diabetes. Biomed Pharmacother. sciencedirect; 2017;92:707-19.

20. Esposito K, Chiodini P, Maiorino MI, Capuano A, Cozzolino D, Petrizzo $\mathrm{M}$, et al. A nomogram to estimate the HbA1c response to different DPP-4 inhibitors in type 2 diabetes: a systematic review and meta-analysis of 98 trials with 24163 patients. BMJ Open. 2015 Feb 1;5(2):e005892.

21. Godoy J G, Gutiérrez V, Montecinos M, Yenes A. Safety and efficacy of Vildagliptin in real life Chilean diabetic patients. Rev Med Chil. 2015 Jan 4;143(1):63-8.

22. Mathieu, Barnett, Brath, Conget, Castro, Göke, et al. Effectiveness and tolerability of second-line therapy with vildagliptin vs. other oral agents in type 2 diabetes: A real-life worldwide observational study (EDGE). International Journal of Clinical Practice. 2013; 67(10):947-56.

23. Scirica B, Bhatt D, Braunwald E, Steg G, Davidson J, Hirshberg B, et al. Saxagliptin and Cardiovascular Outcomes in Patients with Type 2 Diabetes Mellitus. The New England Journal of Medicine. 2013;369(14):1317-26.

24. Green J, Bethel A, Armstrong P, Buse J, Engel S, Garg J, et al. Effect of Sitagliptin on Cardiovascular Outcomes in Type 2 Diabetes. New Engl J Medicine. nejm; 2015;373(3):232-42.

25. Zannad F, Cannon C, Cushman W, Bakris G, Menon V, Perez A, et al. Heart failure and mortality outcomes in patients with type 2 diabetes taking alogliptin versus placebo in EXAMINE: a multicentre, randomised, double-blind trial. Lancet. Sciencedirect; 2015; 385(9982):2067-76.
26. Rosenstock J, Perkovic V, Alexander JH, Cooper ME, Marx N, Pencina MJ, et al. Rationale, design, and baseline characteristics of the CArdiovascular safety and Renal Microvascular outcomE study with LINAgliptin (CARMELINA®): a randomized, double-blind, placebo-controlled clinical trial in patients with type 2 diabetes and high cardio-renal risk. Cardiovasc Diabetol. 2018 Mar 3; 17(1):39.

27. McMurray J, Ponikowski P, Bolli G, Lukashevich V, Kozlovski P, Kothny W, et al. Effects of Vildagliptin on Ventricular Function in Patients With Type 2 Diabetes Mellitus and Heart Failure A Randomized Placebo-Controlled Trial. Jacc Hear Fail. Sciencedirect; 2018; 6(1):8-17.

28. Elgendy I, Mahmoud A, Barakat A, Elgendy A, Saad M, Abuzaid A, et al. Cardiovascular Safety of Dipeptidyl-Peptidase IV Inhibitors: A Meta-Analysis of Placebo-Controlled Randomized Trials. Am J Cardiovasc Drug. Springer; 2017; 17(2):143-55.

29. Fadini G, Saragoni S, Russo P, Esposti L, Kreutzenberg S de, Melazzini $\mathrm{M}$, et al. Intraclass differences in the risk of hospitalization for heart failure among patients with type 2 diabetes initiating a dipeptidyl peptidase- 4 inhibitor or a sulphonylurea: Results from the OsMed Health-DB registry. Diabetes, Obesity and Metabolism. Diabetes, Obesity and Metabolism; 2017;19(10):1416-24.

30. Guo W-QQ, Li L, Su Q, Dai W-RR, Ye Z-LL. Effect of Dipeptidyl Peptidase-4 Inhibitors on Heart Failure: A Network Meta-Analysis. Value Health. 2017 Dec 5;20(10):1427-30.

31. Williams R, de Vries F, Kothny W, Serban C, Lopez-Leon S, Chu C, et al. Cardiovascular safety of vildagliptin in patients with type 2 diabetes: A European multi-database, non-interventional post-authorization safety study. Diabetes, Obesity and Metabolism. 2017; 19(10):1473-8.

32. Bekiari E, Rizava C, Athanasiadou E, Papatheodorou K, Liakos A, Karagiannis $\mathrm{T}$, et al. Systematic review and meta-analysis of vildagliptin for treatment of type 2 diabetes. Endocrine. 2016;52(3):458-80.

33. Filion KB, Azoulay L, Platt RW, of ... D-M. A multicenter observational study of incretin-based drugs and heart failure. New Engl J of Med. 2016.

34. Kongwatcharapong, Dilokthornsakul, Nathisuwan, Phrommintikul, Chaiyakunapruk. Effect of dipeptidyl peptidase-4 inhibitors on heart failure: A meta-analysis of randomized clinical trials. International Journal of Cardiology. 2016;88-95.

35. Sato A, Yoshihisa A, Kanno Y, Takiguchi M, Miura S, Shimizu T, et al. Associations of dipeptidyl peptidase-4 inhibitors with mortality in hospitalized heart failure patients with diabetes mellitus. ESC Heart Fail. 2016 Jun 3;3(2):77-85.

36. McInnes G, Evans M, Prato S, Stumvoll M, Schweizer A, Lukashevich V, et al. Cardiovascular and heart failure safety profile of vildagliptin: a metaanalysis of 17000 patients. Diabetes Obes Metabolism. 2015;17(11):108592.

37. Adler AI, Stevens RJ, Manley SE, Bilous RW, Cull CA, Holman RR, UKPDS GROUP. Development and progression of nephropathy in type 2 diabetes: the United Kingdom Prospective Diabetes Study (UKPDS 64). Kidney Int. 2003;63(1):225-32.

38. So WY, Kong AP, Ma RC, et al. glomerular fltration rate, cardiorenal end points, and all-cause mortality in type 2 diabetic patients. Diabetes Care. 2006;29(9):2046-52.

39. Ninomiya T, Perkovic V, de Galan BE, et al. Albu minuria and kidney function independently predict cardiovascular and renal outcomes in diabetes. J Am Soc Nephrol. 2009;20(8):1813-21. 
40. Johnston SS, Conner C, Aagren M, Smith DM, Bouchard J, Brett J. Evidence linking hypoglycemic events to an increased risk of acute cardiovascular events in patients with type 2 diabetes. Diabetes Care. 2011;34(5):1164-70.

41. Gerstein HC, Miller ME, Byington RP, Goff DC, Bigger JT, Buse JB, et al. Effects of intensive glucose lowering in type 2 diabetes. $N$ Engl $\mathrm{J}$ Med. 2008 Jun 4; 358(24):2545-59.

42. Ismail-Beigi F, Craven T, Banerji MA, et al. Effect of intensive treatment of hyperglycaemia on microvascular outcomes in type 2 diabetes: an analysis of the ACCORD randomised trial. Lancet. 2010; 376(9739):419-30.

43. Howse P, Chibrikova L, Twells L, Barrett B, Gamble J-M. Safety and Efficacy of Incretin-Based Therapies in Patients With Type 2 Diabetes Mellitus and CKD: A Systematic Review and Meta-analysis. Am J Kidney Dis. sciencedirect; 2016;68(5):733-42.

44. Thomas MC, Paldánius PM, Ayyagari R, Ong S, Groop P-H. Systematic Literature Review of DPP-4 Inhibitors in Patients with Type 2 Diabetes Mellitus and Renal Impairment. Diabetes Therapy. Diabetes Therapy; 439-54.

45. Kothny W, Lukashevich V, Foley J, Rendell M, Schweizer A. Comparison of vildagliptin and sitagliptin in patients with type 2 diabetes and severe renal impairment: a randomised clinical trial. Diabetologia. 2015;58(9):2020-6.

46. Fonseca V, Gill J, Zhou R, Leahy J. An analysis of early insulin glargine added to metformin with or without sulfonylurea: impact on glycaemic control and hypoglycaemia. Diabetes Obes Metab. 2011;13(9):814-822.

47. Aschner P, Chan J, Owens DR, et al. EASIE Investigators. Insulin glargine versus sitagliptin in insulin-naive patients with type 2 diabetes mellitus uncontrolled on metformin (EASIE): a multicentre, randomised open-label trial. Lancet. 2012;379(9833):2262-2269.

48. Action to Control Cardiovascular Risk in Diabetes Study Group, Gerstein HC, Miller ME, Byington RP, Goff Jr DC, Bigger JT, Buse JB, et al. Effects of intensive glucose lowering in type 2 diabetes. N Engl J Med 2008;358:2545-59.

49. Tsukube, S., Ikeda, Y., Kadowaki, T., and Odawara, M. (2015) Improved Treatment Satisfaction and Self-reported Health Status after Introduction of Basal-Supported Oral Therapy Using Insulin Glargine in Patients with Type 2 Diabetes: Sub-Analysis of ALOHA2 Study, Diabetes Therapy 6, 153-171.

50. Jabbour S. Primary care physicians and insulin initiation: multiple bar riers, lack of knowledge or both? Int J Clin Pract. 2008; 62:845-847.

51. Kanazawa I, Tanaka K, Notsu M, Tanaka S, Kiyohara N, Koike S, et al. Long-term efficacy and safety of vildagliptin add-on therapy in type 2 diabetes mellitus with insulin treatment. Diabetes Res Clin Pr. Sciencedirect; 2017; 123:9-17.

52. Li F-FF, Shen Y, Sun R, Zhang D-FF, Jin X, Zhai X-FF, et al. Effects of Vildagliptin Add-on Insulin Therapy on Nocturnal Glycemic Variations in Uncontrolled Type 2 Diabetes. Diabetes Ther. 2017 Oct;8(5):1111-22.

53. Gautier JF, Monguillon P, Verier-Mine O, Valensi P, Fiquet B, Dejager S, et al. Which oral antidiabetic drug to combine with metformin to minimize the risk of hypoglycemia when initiating basal insulin? A randomized controlled trial of a DPP4 inhibitor versus insulin secretagogues. Diabetes Res Clin Pr. 2016;116:26-8.

54. Kim Y, Min S, Hahn S, Oh T, Park K, Cho Y. Efficacy and safety of the addition of a dipeptidyl peptidase-4 inhibitor to insulin therapy in patients with type 2 diabetes: A systematic review and meta-analysis. Diabetes Research and Clinical Practice. 2016;116:86-95.
55. Chen C, Yu Q, Zhang S, Yang P, Wang C-Y. Assessing the efficacy and safety of combined DPP-4 inhibitor and insulin treatment in patients with type 2 diabetes: a meta-analysis. Int J Clin Exp Patho. 2015;8(11):14141-50.

56. Ito D, Inoue K, Kaneko K, Yanagisawa M, Sumita T, Ikegami Y, et al. The efficacy of vildagliptin concomitant with insulin therapy in type 2 diabetic subjects. J Clin Med Res. 2015 May 5;7 (5):303-7.

57. Thomas MC, Cooper ME, Zimmet P..Changing epidemiology of type 2 diabetes mellitus and associated chronic kidney disease. Nat Rev Nephrol. 2016 Feb;12 (2):73-81.

58. Fox CS, Matsushita K, Woodward M, et al, for the Chronic Kidney Disease Prognosis Consortium. Associations of kidney disease measures with mortality and end-stage renal disease in individuals with and without diabetes: A meta-analysis of 1024977 individuals. Lancet 2012; 380: 1662-73.

59. Nauck MA, Meier JJ, Cavender MA, Abd El Aziz M, Drucker DJ. Cardiovascular Actions and Clinical Outcomes With Glucagon-Like Peptide-1 Receptor Agonists and Dipeptidyl Peptidase-4 Inhibitors. Circulation. 2017 Aug 2;136(9):849-70.

\section{Abreviaturas}

add-on: en combinación.

DE: Desviación estándar

DBT: Diabetes

DM2: Diabetes Mellitus tipo 2

DPP-4: Enzima Dipeptidil-Peptidasa-4

DPP-4I: Inhibidor de dipeptidil peptidasa 4

ECCA: Ensayo Clínico Controlado Asignado Aleatoriamente

GLP-1: Glucagón péptido 1

GIP: Péptido inhibidor gástrico GIP

HBA1c: Hemoglobina glicosilada A1c

IC: Intervalo de confianza

OR: Odss ratio

$r$ : Coeficiente de correlación 